\title{
A Rare Case of Severe Copper Deficiency in an Infant with Exclusive Breast Feeding Mimicking Myelodysplastic Syndrome
}

\author{
Samah Kohlaa, b Elrazi Alic ${ }^{c}$ Aliaa Amer $^{a}$ Tayseer Yousif ${ }^{d}$ \\ Mohamed A. Yassine \\ aDepartment of Laboratory Medicine and Pathology, Hematology Section, Hamad Medical \\ Corporation, Doha, Qatar; ${ }^{b}$ Clinical Pathology Department, Hematology Section, Faculty \\ of Medicine/ Al-Azhar University, Cairo, Egypt; 'Department of Internal Medicine, Hamad \\ Medical Corporation, Doha, Qatar; ${ }^{\mathrm{d} D e p a r t m e n t}$ of Pediatric Hematology and Oncology, \\ Hamad Medical Corporation, Doha, Qatar; ${ }^{e}$ Department of Hematology, Hamad Medical \\ Corporation, Doha, Qatar
}

\section{Keywords}

Anemia $\cdot$ Breast feeding $\cdot$ Copper deficiency $\cdot$ Myelodysplastic syndrome $\cdot$ Ringed sideroblasts

\begin{abstract}
An 11-month-old full-term female infant was referred to the hematology clinic due to marked anemia and neutropenia. She was almost exclusively breastfed and rejecting all trials for supplementary food including artificial formulas. Bone marrow aspirate revealed cytoplasmic vacuolization in precursors of the myeloid and erythroid series with significant dysgranulopoiesis and dyserythropoiesis and ringed sideroblasts. Flow cytometry analysis revealed increased hematogones with aberrant loss/downregulation of CD33 on granulocytes and monocytes (sign of dysmyelopoiesis). Laboratory investigation revealed low serum copper and ceruloplasmin. Administration of a multivitamin including a high concentration of copper for only 1 week improved her hemoglobin and absolute neutrophil count up to $1.9 \times 103 / \mu \mathrm{L}$, then dropped to $0.3103 / \mu \mathrm{L}$ after she stopped taking the copper multivitamin. Her blood counts improved till total normalization and up to the time this report is issued. The probable role of unrecognized copper deficiency in causing anemia in infants more than 6 months of age is discussed, and the importance of serum copper examination in refractory anemia and neutropenia is emphasized. This case shows that copper deficiency should be an integral part of the differential diagnosis of refractory anemia including sideroblastic anemia and dysplasia. To the best of our knowledge, no such case has previously been described in the literature.
\end{abstract}


Kohla et al.: Severe Copper Deficiency in an Infant with Exclusive Breast Feeding Mimicking MDS

\section{Introduction}

Copper is a trace microelement that plays a pivotal role in many enzymatic reactions at the cellular level. Impaired function of copper-dependent enzymes can explain some of the clinical manifestations of copper deficiency like muscle weakness (cytochrome $c$ oxidase), lack of skin pigmentation (dopamine beta-hydrolase) and bleeding tendency (decreased factor V) [1-13]. The recommended daily allowance for copper is low for both adults and children $(24 \mu \mathrm{g} / \mathrm{kg} /$ day in children aged 6-12 months). Therefore, copper deficiency is not common unless there is a predisposing factor, for example chronic diarrhea or malabsorption, foregut surgery [2], prolonged parenteral nutrition, excess zinc intake [3] or hereditary disorders like Menkes disease [4].

Copper deficiency can lead to several hematological manifestations including anemia, which usually is microcytic but can also be normocytic or macrocytic, neutropenia and, less commonly, thrombocytopenia [5]. Copper deficiency can also present as sideroblastic anemia and may even mimic myelodysplastic syndrome [6]. The latter is predominantly a disease of adults but is occasionally seen in the pediatric age group [7], though the presence of this condition should raise the suspicion of other mimics which carry a more favorable prognosis than copper deficiency.

Clinically, the diagnosis of copper deficiency is made by low serum copper, supported by low serum ceruloplasmin, excluding other causes which can give a similar presentation, such as $\mathrm{B}_{12}$ deficiency [8].

\section{Case Report}

We present the case of a 2-year-old girl who was referred to the hematology clinic at the age of 11 months due to marked anemia (macrocytic for age) with a hemoglobin ( $\mathrm{Hb}$ ) level of $6.3 \mathrm{~g} / \mathrm{dL}$ and marked neutropenia with an absolute neutrophil count (ANC) of $0.1 \times 10^{3} / \mu \mathrm{L}$. Neutropenia was found mostly since birth with an ANC of $1.1 \times 10^{3} / \mu \mathrm{L}$ at the age of 1 month; then no other readings were recorded till the age of 11 months after which all readings were below $0.3 \times 10^{3} / \mu \mathrm{L}$. Reticulocyte count ranged between 56.5 and $137 \times 10^{3} / \mu \mathrm{L}$. She was born at term (uneventful normal vaginal delivery), had normal weight, but developed vomiting and poor weight gain from early life on. She had gastroesophageal reflux disease grade 2 at the age of 5 weeks (diagnosed with a barium swallow), which improved on treatment. She was almost exclusively breastfed, rejecting all trials for supplementary food including artificial formulas when she was first seen in the clinic. She had developmental delay, abdominal distension, and loose motions. Her immunization was up to date and she had no history of significant infections or recurrent use of antibiotics or gut surgery. On physical examination, her weight was $7.7 \mathrm{~kg}$ (below centiles). There were no dysmorphic features; she had a fair complexion (looks fairer than her parents) and thin soft hair. Her liver was slightly enlarged with a span of $10 \mathrm{~cm}$. No splenomegaly and no palpable lymph nodes were found. Mild hypotonia was reported, but the rest of the systemic examination was unremarkable. Abdominal ultrasound showed mild hepatomegaly with a diffuse fatty change in the hepatic parenchyma. Her laboratory findings revealed negative stool analysis for ova and parasites and negative allergy testing for egg, milk and gluten sensitivity. Hb electrophoresis was normal for her age. Viral serology for hepatitis A, B and C, varicella, CMV and parvovirus B19 (IgG, IgM) were negative. Folate, vitamin $\mathrm{B}_{12}$, TSH, T3, T4 and insulin levels were all normal. Iron panel was normal apart from low iron saturation of $5.0 \%$ (15-45\%). Her CBC showed marked anemia with $\mathrm{Hb}$ of $5.5 \mathrm{~g} / \mathrm{dL}$, WBCs of $10.9 \times 10^{3} / \mu \mathrm{L}$, platelets of $1,131 \times 10^{3} / \mu \mathrm{L}$, ANC of $0.1 \times 10^{3} / \mu \mathrm{L}$ and increased absolute reticulocyte count of $134.1 \times 10^{9} / \mathrm{L}$. The patient was scheduled for bone marrow examination. 
Kohla et al:: Severe Copper Deficiency in an Infant with Exclusive Breast Feeding Mimicking MDS

Fig. 1. Peripheral blood smear shows macrocytic anemia with a dimorphic picture (RBC morphologic features are evident with a population of microcytic/hypochromic erythrocytes), polychromasia, marked neutropenia and marked thrombocytosis (Wright-Giemsa. $\times 500)$.

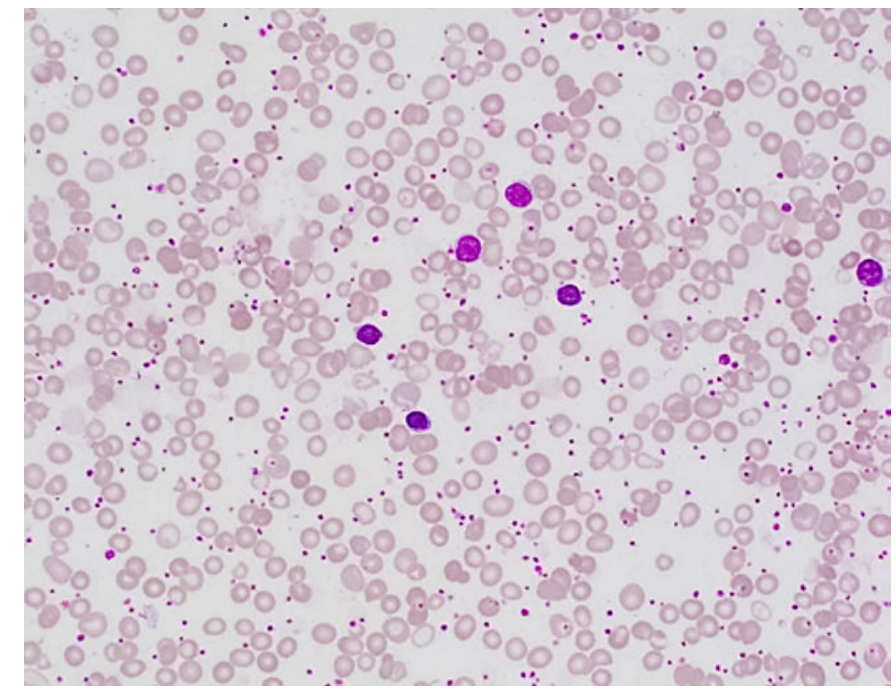

Peripheral blood smear showed marked macrocytic anemia with a dimorphic picture, polychromasia, marked neutropenia and marked thrombocytosis (Fig. 1).

Bone marrow aspirate showed increased megakaryocytes with clusters, with small and/ or hypolobated forms noted and the smear was stuffed with large pools of platelets in the background (Fig. 2). There were 3\% blasts and prominent cytoplasmic vacuolization in granulocytic precursors (mainly in the promyelocyte and myelocyte stages), in eosinophils and in early erythroid precursors. Granulopoiesis was left shifted with significant dysgranulopoietic changes (hypo/non-segmentation and abnormal segmentation). Erythropoiesis was hyperplastic (M/E 0.5) with significant dyserythropoietic changes (megaloblastoid maturation, karyorrhexis, budding, binuclearity and rare internuclear bridging) (Fig. 3).

Iron in stores was adequate with increased sideroblasts and ringed sideroblasts (9\%) (Fig. 4).

Bone marrow trephine biopsy was normocellular for her age (95-100\%) with trilineage hematopoiesis, increased early erythroid precursors and increased megakaryocytes. There was no increase in CD34-positive cells by immunohistochemical stains.

Flow cytometry analysis on bone marrow aspirate showed 2\% CD34-positive myeloblasts, $18 \%$ T-cells and 13\% B-cells with no immunophenotypic evidence of monotypic B-cell population. There were $6 \%$ CD19/CD10-positive cells with partial expression of CD20 and CD34 (representing hematogone hyperplasia). Cells in the granulocytic gate comprise $25 \%$ expressing CD15, CD13, CD11b, and CD16 with aberrant loss of CD33. Cells in the monocytic gate comprise $7 \%$ showing dim expression of CD33. Aberrant loss/downregulation of CD33 on granulocytes or on monocytes might be a sign of dysmyelopoiesis.

Cytogenetic analysis revealed normal female karyotype: 46,XX. Further laboratory testing revealed a low serum copper level $(<7.0 \mu \mathrm{mol} / \mathrm{L}[14.1-29.8 \mu \mathrm{mol} / \mathrm{L}])$ and a low serum zinc level $(8.51 \mu \mathrm{mol} / \mathrm{L}$ [9.18-18.36 $\mu \mathrm{mol} / \mathrm{L}])$ on repeated measurements and a low ceruloplasmin level $(2.5 \mathrm{mg} / \mathrm{dL}[18.0-37.0 \mathrm{mg} / \mathrm{dL}])$.

Fecal elastase was normal initially, but the subsequent readings were low. Sweat chloride test was normal. Limited genetic panel for Wilson's disease was normal.

Copper extract was not available, so she was started on a multivitamin supplement including a high concentration of copper. She took the medicine for only 1 week and rejected it later. Two weeks later, her ANC was improved up to $1.9 \times 10^{3} / \mu \mathrm{L}$. After that, she stopped 
Kohla et al.: Severe Copper Deficiency in an Infant with Exclusive Breast Feeding Mimicking MDS

Fig. 2. Bone marrow aspirate. A Increased megakaryocytes with cluster formation and large pool of platelets (Wright-Giemsa. ×200). B Small and hypolobated megakaryocytes (WrightGiemsa. ×1,000).

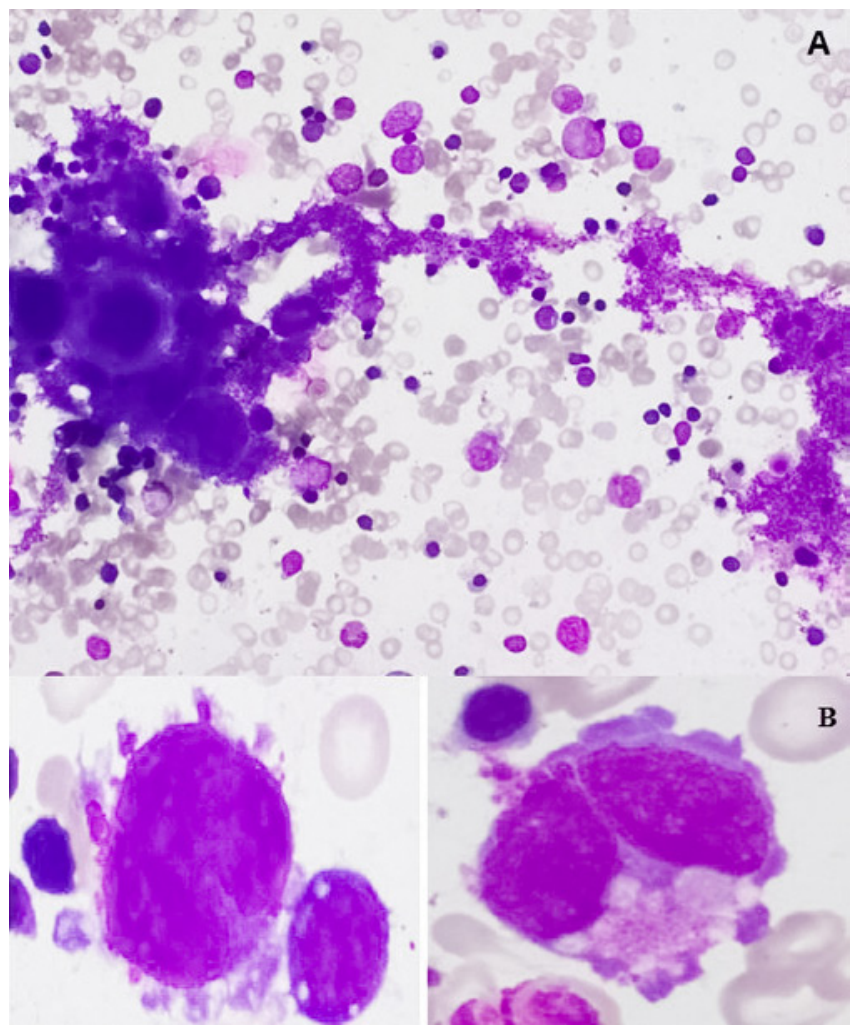

Fig. 3. Bone marrow morphologic changes in copper deficiency. A Prominent cytoplasmic vacuolization in granulocytic precursors, eosinophils and in early erythroid precursors, left-shifted granulopoiesis with little maturation beyond the myelocyte stage, significant dysgranulopoietic features (hypo/non-segmentation and abnormal segmentation) (Wright-Giemsa. ×500). B Increased erythropoiesis with dyserythropoietic features (megaloblastoid changes, karyorrhexis, budding, binuclearity, internuclear bridging) (Wright-Giemsa. ×500).

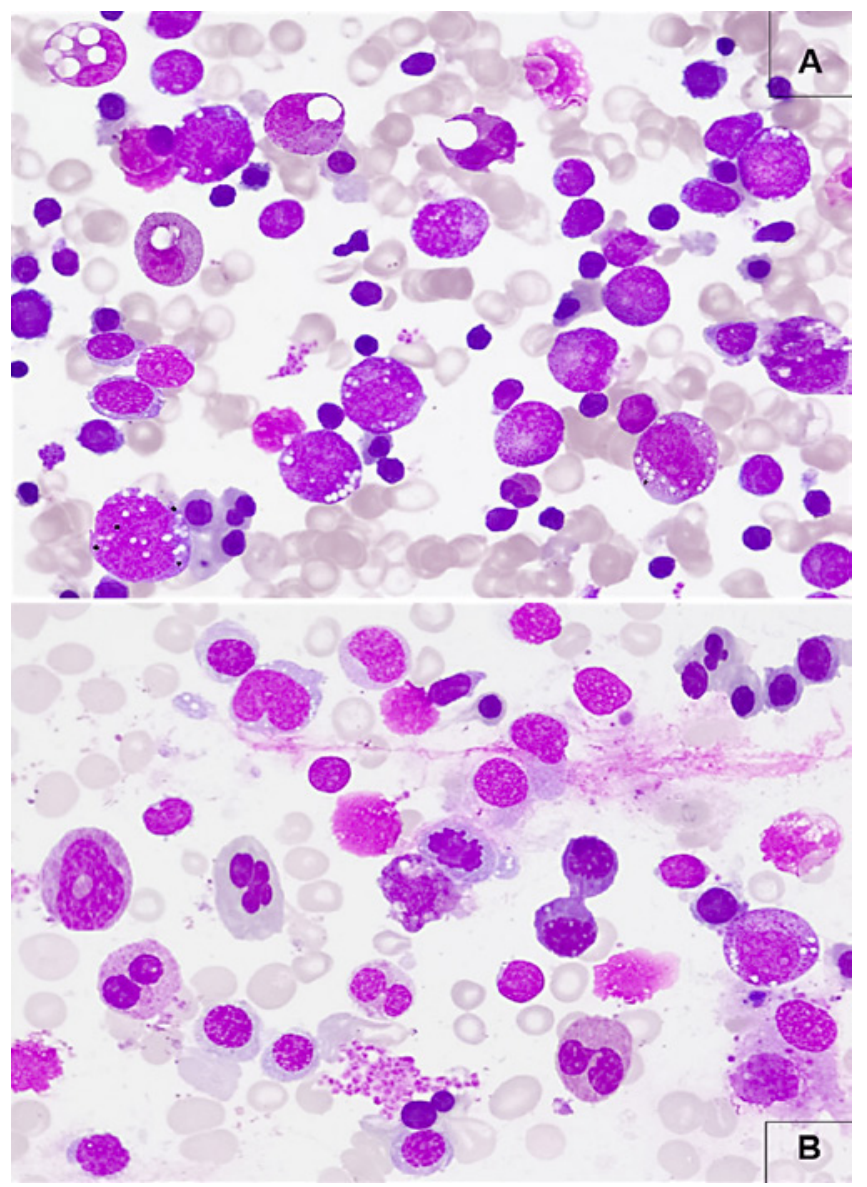


Kohla et al.: Severe Copper Deficiency in an Infant with Exclusive Breast Feeding Mimicking MDS

Fig. 4. Iron stain (composite) increased sideroblasts and ringed sideroblasts 9\% (Prussian blue. $\times 1,000$ ).

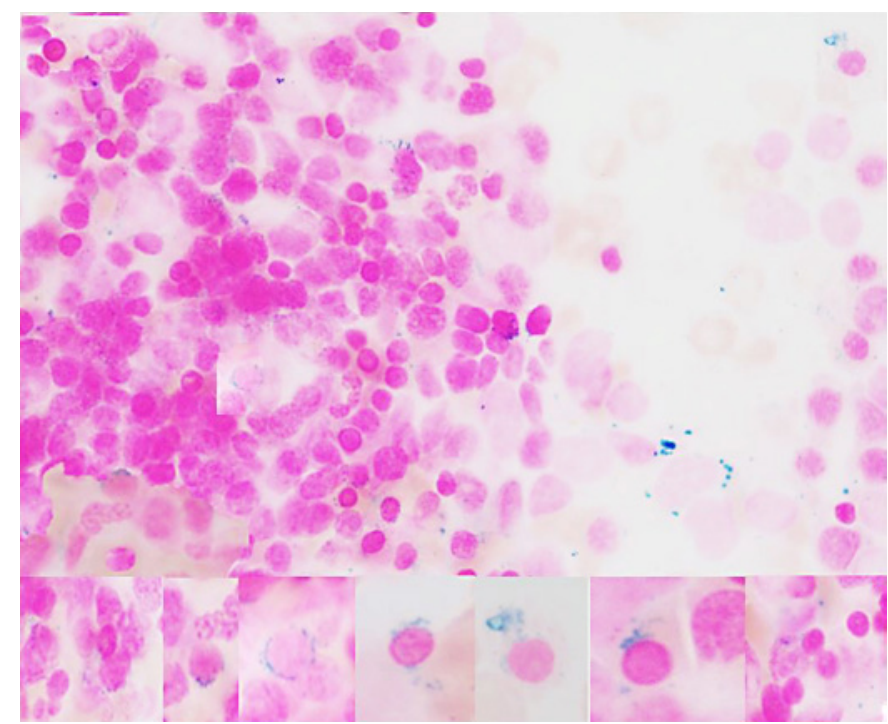

taking the copper-multivitamin syrup and her ANC dropped again to $0.3 \times 10^{3} / \mu \mathrm{L}$. For the next 3 months, her blood counts improved till total normalization.

She did not have recurrent infections and continued to have intermittent loose motions with occasional undigested food particles. No dermatitis or itching was seen; she was attending developmental milestones, with speech slightly slow but good comprehension and hearing.

She was also weaned gradually with the introduction of supplementary food.

\section{Discussion}

Cytopenias with macrocytosis and vacuolated hematopoietic precursors in the bone marrow were suggestive of copper deficiency (whether congenital or acquired), which also results in a myelodysplastic syndrome-like picture and ringed sideroblasts, associated with a low level of ceruloplasmin, failure to thrive, food intolerance and vomiting, especially when the patient was on exclusive breastfeeding. The thrombocytosis noted in this case was transient and seemed to be reactive in origin. Copper deficiency has been reported to result in anemia, neutropenia and less commonly, thrombocytopenia. Unlike myelodysplastic syndrome, the bone marrow aspirate in copper deficiency characteristically shows cytoplasmic vacuoles within erythroid and myeloid precursors. Other abnormalities include megaloblastic changes and ringed sideroblasts [9]. Furthermore, karyotyping in cases of copper deficiency does not reveal cytogenetic features characteristic of myelodysplastic syndrome.

This girl with bicytopenia since the neonatal period and failure to thrive was found to have macrocytic anemia and neutropenia and bone marrow examination coping with copper deficiency with no apparent precipitating factors. It might be explained by dietary deficiency as the patient was almost exclusively breastfed, not tolerating milk formula or other feeds. Since human breast milk contains a considerably small amount of copper $[10,11]$, this amount drops significantly from the early period of lactation from around $0.7 \mathrm{mg} / \mathrm{L}$ to $0.2 \mathrm{mg} / \mathrm{L}$ and may provide less than the copper intake recommended by the World Health Organization (WHO) [4]. During this period, any shortfall in copper intake can be met by mobilizing liver copper stores. However, on several occasions, there is no predisposing factor identified causing copper deficiency [12]. The patient's zinc level was repeatedly low, and thus zinc 
Kohla et al.: Severe Copper Deficiency in an Infant with Exclusive Breast Feeding Mimicking MDS

toxicity as a cause for her copper deficiency was excluded. She also did not have any features suggestive of Menkes disease and no frank picture of malabsorption syndromes including negative celiac screen.

Our patient did not have any significant radiation or toxic exposure to our knowledge.

Clinically the patient was fairer than her patients, which may be a feature of congenital copper deficiency due to decreased dopamine beta-hydrolase.

Pearson marrow-pancreas syndrome, a severe disorder that usually begins in infancy and is considered a bone marrow failure disorder, could be another possibility. In people with Pearson marrow-pancreas syndrome, the pancreas is also affected. It is generally not inherited but arises from new (de novo) mutations that likely occur in early embryonic development [13]. Pearson marrow disease was excluded in our patient given the normal level of elastase and the negative genetic tests.

The low serum copper and the response to therapeutic challenge to copper as well as the patient's karyotype were normal $(46, \mathrm{XX})$, and there were intracytoplasmic vacuolations, which go with copper deficiency; moreover, ringed sideroblasts support the copper deficiency over myelodysplasia.

Additionally, there are approximately $6 \%$ hematogones at different stages of maturation. This hematogone hyperplasia is almost absent in myelodysplastic syndrome, and suggests nonclonal cytopenia, such as copper deficiency.

Nutritional copper deficiency remains to be a highly possible cause for this infant's picture supported by the rapid response to oral copper supplement with normalization of blood counts, then reversal of that response when she was off therapy and regain of the normal counts perhaps due to introduction of less breastfeeding and introduction of food supplements thereafter.

\section{Conclusion}

In patients with cytopenia or low-grade myelodysplasia, copper deficiency should be suspected even if there is no clear predisposing risk factor for copper deficiency, and a trial of copper treatment, if the copper level is low, is reasonable as it is a treatable condition.

\section{Acknowledgement}

The authors would like to acknowledge Qatar national library and co-workers, without whom this work would not have been possible.

\section{Statement of Ethics}

The authors have no ethical conflicts to disclose. The case report was conducted ethically in accordance with the guideline of the Medical Research Center (MRC) of Hamad Medical Corporation (HMC), and after obtaining their approval. Informed consent was provided by the patient to publish the case.

\section{Disclosure Statement}

The authors declare that they have no relevant financial interests. 
Kohla et al.: Severe Copper Deficiency in an Infant with Exclusive Breast Feeding Mimicking MDS

\section{Funding Sources}

Qatar National library funded the study.

\section{Authors Contributions}

Dr. Samah Kohla did the literature review and wrote the hematology part of the manuscript. Dr. Elrazi Ali, Tayseer Yousif and Mohamed A. Yassin wrote the clinical part of the manuscript. Dr. Aliaa Amer reviewed the hematology part of the manuscript.

\section{References}

1 Prohaska JR. Biochemical changes in copper deficiency. The J Nutr Biochem. 1990;1:452-61.

2 Kumar N, Ahlskog JE, Gross JB. Acquired hypocupremia after gastric surgery. Clin Gastroenterol Hepatol. 2004 Dec;2(12):1074-9.

3 Rowin J, Lewis SL. Copper deficiency myeloneuropathy and pancytopenia secondary to overuse of zinc supplementation. J Neurol Neurosurg Psychiatry. 2005 May;76(5):750-1.

4 Ashkenazi A, Levin S, Djaldetti M, Fishel E, Benvenisti D. The syndrome of neonatal copper deficiency. Pediatrics. 1973 Oct;52(4):525-33.

5 Halfdanarson TR, Kumar N, Li C-Y, Phyliky RL, Hogan WJ. Hematological manifestations of copper deficiency: a retrospective review. Eur J Haematol. 2008 Jun;80(6):523-31.

6 Huff JD, Keung YK, Thakuri M, Beaty MW, Hurd DD, Owen J. Copper deficiency causes reversible myelodysplasia. Am J Heamtol. 2007 Jul;82(7):625-30.

7 Hasle H. Myelodysplastic syndromes in childhood-classification, epidemiology, and treatment. Leuk Lymphoma. 1994;13(1-2):11-26.

8 Halfdanarson TR, Kumar N, Li CY, Phyliky RL, Hogan WJ. Hematological manifestations of copper deficiency: a retrospective review. Eur J Haematol. 2008;80:523-31.

9 D’Angelo G. Copper deficiency mimicking myelodysplastic syndrome. Blood Res. 2016 Dec;51(4):217-9.

10 Lönnerdal B. Copper nutrition during infancy and childhood. Am J Clin Nutr. 1998 May;67(5 Suppl):1046S$53 \mathrm{~S}$.

11 Vuori E. Intake of copper, iron, manganese and zinc by healthy, exclusively-breast-fed infants during the first 3 months of life. Br J Nutr. 1979 Nov;42(3):407-11.

12 Harless W, Crowell E, Abraham J. Anemia and neutropenia associated with copper deficiency of unclear etiology. Am J Hematol. 2006 Jul;81(7):546-9.

13 Manea EM, Leverger G, Bellmann F, Stanescu PA, Mircea A, Lèbre AS. Pearson syndrome in the neonatal period: two case reports and review of the literature. J Pediatr Hematol Oncol. 2009 Dec;31(12):947-51. 